\title{
Semi-Markovian Model of Unreliable Control of Restorable System with Latent Failures
}

\author{
Yuriy E. Obzherin, Aleksei I. Peschansky, Yelena G. Boyko \\ Sevastopol National Technical University, Sevastopol, Ukraine \\ E-mail:vmsevntu@mail.ru \\ Received December 27, 2010; revised January 3, 2011; accepted January 5, 2011
}

\begin{abstract}
Semi-Markovian model of control of restorable system with latent failures has been built with regard to control errors. Stationary reliability and efficiency characteristics of its operation have been found. The problem of control execution periodicity optimization has been solved.
\end{abstract}

Keywords: Control, Control Errors, Latent Failure, Semi-Markovian Process, System Stationary Characteristics

\section{Introduction}

An important factor providing reliability, high quality, and efficiency of technological complexes is the presence of control systems in them. The reviews of the results concerning control model building are contained in $[1,2]$.

In the present article the model of control execution and restoration of a single-unit system with latent failures has been investigated under the condition of control errors occurrence. The latent failure is the one that does not show up till the control is executed. While control is executed errors of first and second kinds are possible [3]. It reduces technological complex operation efficiency.

In the work [4] the model of control of the system with possible failures was studied, but there reliability characteristics were defined under the assumption of exponential distributions of random values characterizing the system.

The problems of technological complexes' control are closely connected with their maintenance. In the work [5] maintenance models were built by means of semiMarkovian processes with a common phase field of states [6]. In the present article this apparatus is used to build the model of control under the condition of latent failures occurrence. And herewith, random values characterizing the system are supposed to have distributions of general kind.

\section{The Problem Definition and Mathematical Model Building}

Let us investigate the system operating in the following way. At the time zero the system begins operating, and the control is on. System failure-free operation time is a random value (RV) $\alpha$ with distribution function (DF) $F(t)=P\{\alpha \leq t\}$ and distribution density (DD) $f(t)$. The control is executed in random time $\delta$ with $\mathrm{DF}$ $R(t)=P\{\delta \leq t\}$ and DD $r(t)$. The failure is detected only when control is carried out. Control duration is RV $\gamma$ with DF $V(t)=P\{\gamma \leq t\}$ and DD $v(t)$. When the control is on, the system does not operate. After failure detection system restoration begins immediately and the control is deactivated. System restoration time is RV $\beta$ with DF $G(t)=P\{\beta \leq t\}$ and DD $g(t)$. After the system restoration all its properties are completely restored. All the RV are supposed to be independent, have finite assembly averages and variances.

While control execution errors of first and second kinds can take place. Control system error of first kind lies in regarding system in up state as a failed one (false failure). The probability of such an errror is equal to $p_{1}$. The error of second kind is taking system's good state for bad one (failure omission). The probability of such an error is equal to $\mathrm{p}_{0}$.

The purpose of the present article is to find stationary reliability and economical characteristics of the single-unit restorable system under the condition of latent failures occurrence, with regard to control errors.

To describe the system operation let us use semiMarkovian process $\xi(t)$ with the following field of states:

$$
E=\{111,212 x, 211 x, 101 x, 202,201,200,210\},
$$


where

111 means the system has been restored, the control is activated;

$212 x$ - control has begun, the system is in up state, it does not operate during control execution, time $x$ is left till the latent failure (regardless of deactivation time);

$211 x$ - control has ended, the system in up state has been treated correctly and continues to operate, time $x$ is left till the latent failure;

$101 x$ - latent failure has occurred, time $x$ is left till control execution;

202 - control has begun, the system is in a state of latent failure, it does not operate;

201 - control has ended, failed system is regarded as the one in up state (error of second kind), its operation has begun;

200 - control has ended, failed system is regarded as a failed one, system restoration has begun, control is deactivated;

210 - control has ended, system in up state is taken for failed one (error of first kind), system restoration has begun, control is deactivated;

Time diagram of the system operation is represented in the Figure 1. And in the Figure 2 the system transition graph is given, where $E_{+}$a set of up states is, $E_{-}$is a set of down states.

Time diagram of the system operation and the system transition graph are shown in Figure $\mathbf{1}$ and Figure 2 respectively.

Let us define the probabilities of the embedded Markovian chain (EMC) $\left\{\xi_{n}, n \geq 0\right\}$ transitions:

$$
\begin{gathered}
p_{111}^{212 x}=\int_{0}^{\infty} f(x+t) r(t) d t, x>0 ; \\
p_{111}^{101 x}=\int_{0}^{\infty} r(x+t) f(t) d t, x>0 ; \\
p_{211 x}^{101 y}=r(x+y), y>0 ; \\
p_{211 x}^{212 y}=r(x-y), 0<y<x ; \\
P_{212 x}^{211 x}=\bar{p}_{1}, P_{212 x}^{210}=p_{1} ; \\
P_{101 x}^{202}=P_{201}^{202}=P_{200}^{111}=P_{210}^{111}=1 ; \\
P_{202}^{201}=p_{0}, \quad P_{202}^{200}=\bar{p}_{0} \quad \bar{p}_{i}=1-p_{i}, i=0,1 .
\end{gathered}
$$

Let us indicate $\rho(111), \rho(202), \rho(201), \rho(210)$, $\rho(200)$ the values of EMC $\left\{\xi_{n}, n \geq 0\right\}$ stationary distribution for the states $111,202,201,210,200$ respectively and assume the existence of stationary densities $\rho(212 x), \rho(211 x), \rho(101 x)$ for the states $212 x$, $211 x, 101 x$ respectively. Now we can make the system of integral equations for them:

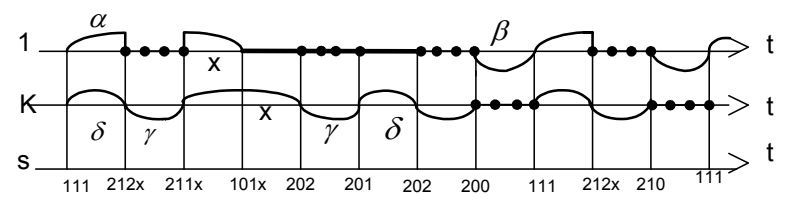

Figure 1. Time diagram of the system operation.

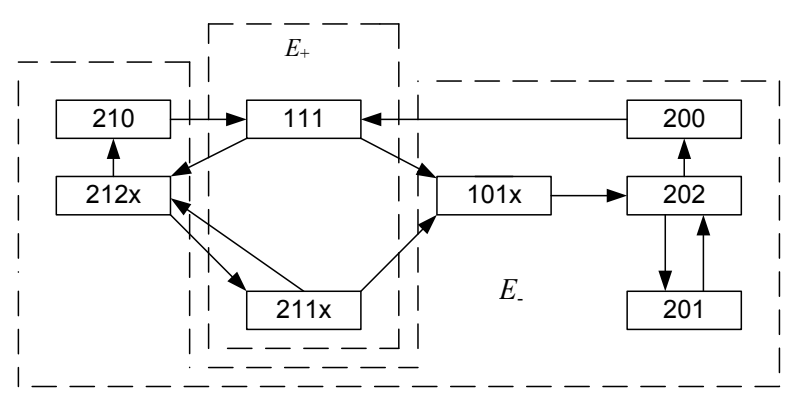

Figure 2. System transition graph.

$$
\left\{\begin{array}{l}
\rho_{0}=\rho(111)=\rho(200)+\rho(210), \\
\rho(212 x)=\rho(111) \int_{0}^{\infty} f(x+t) r(t) d t \\
\quad+\int_{x}^{\infty} \rho(211 y) r(y-x) d y, \\
\rho(211 x)=\bar{p}_{1} \rho(212 x), \\
\rho(101 x)=\rho(111) \int_{0}^{\infty} f(t) r(x+t) d t \\
\quad+\int_{x}^{\infty} \rho(211 y) r(y+x) d y, \\
\rho(202)=\int_{0}^{\infty} \rho(101 x) d x+\rho(201), \\
\rho(201)=p_{0} \rho(202), \\
\rho(200)=\bar{p}_{0} \rho(202), \\
\rho(210)=p_{1} \int_{0}^{\infty} \rho(212 x), \\
2 \rho_{0}^{\infty} \rho(211 x) d x+\int_{0}^{\infty} \rho(101 x) d x=1 .
\end{array}\right.
$$

The last equation of the system (2) is a normalization requirement.

To solve the system (2) let us exclude the function $\rho(211 x)$ from the second and third equations:

$$
\rho(212 x)=\rho_{0} \int_{0}^{\infty} f(x+t) r(t) d t+\bar{p}_{1} \int_{x}^{\infty} \rho(212 y) r(y-x) d y
$$


Let us indicate $\rho(212 x)=\varphi_{1}(x)$, then the Equation (3) will take the form:

$$
\begin{aligned}
\varphi_{1}(x) & =\rho_{0} \int_{x}^{\infty} f(t) r(t-x) d t \\
& +\bar{p}_{1} \int_{x}^{\infty} \varphi_{1}(y) r(y-x) d y
\end{aligned}
$$

The solution of this equation will be defined with the help of method of successive approximations.
Let us introduce the function $\tilde{r}(x)=\bar{p}_{1} \cdot r(x)$ and the integral operator $\left[A_{\tilde{r}} \varphi\right](x)=\int_{x}^{\infty} \tilde{r}(y-x) \varphi(y) d y$. Then the Equation (4) can be rewritten in the following way:

$$
\varphi_{1}=A_{\ddot{r}} \varphi_{1}+\frac{\rho_{0}}{\bar{p}_{1}} A_{\tilde{r}} f .
$$

The solution of this equation is defined by the formula

$$
\rho(212 x)=\varphi_{1}(x)=\rho_{0} \cdot \frac{1}{\bar{p}_{1}} \int_{x}^{\infty} h_{\tilde{r}}(y-x) f(y) d y=\frac{\rho_{0}}{\bar{p}_{1}} \int_{0}^{\infty} h_{\tilde{r}}(y) f(x+y) d y,
$$

where $h_{\tilde{r}}(t)$ is the density of renewal function $H_{\tilde{r}}(t)$ generated by the RV with improper DF $\tilde{R}(t)=\bar{p}_{1} R(t)$; $h_{\tilde{r}}(t)=\sum_{n=1}^{\infty} \tilde{r}^{*(n)}(t)$, where $\tilde{r}^{*(n)}(t)$ is n-fold convolu- tion of the function $\tilde{r}(t)=\bar{p}_{1} \cdot r(t)$.

Using Formula (5), one can define the rest of stationary densities

$$
\rho(211 x)=\rho_{0} \int_{0}^{\infty} h_{\tilde{r}}(y) f(x+y) d y, \quad \rho(101 x)=\frac{\rho_{0}}{\bar{p}_{1}} \int_{0}^{\infty} f(z) \tilde{v}_{r}(z, x) d z,
$$

where $\tilde{v}_{r}(z, x)=\tilde{r}(x+z)+\int_{0}^{z} \tilde{r}(x+y) h_{\tilde{r}}(z-y) d y$ is the density of direct residual time of renewal process gener-

ated by RV with improper distribution density $\tilde{r}(t)$.

The values of stationary distribution for the states 210 , 202, 201, 200 are defined from the system (2):

$$
\begin{aligned}
& \rho(210)=\rho_{0} \frac{p_{1}}{\bar{p}_{1}} \int_{0}^{\infty} H_{\tilde{r}}(z) f(z) d z, \quad \rho(202)=\rho_{0} \frac{1}{\bar{p}_{0}}\left(1-\frac{p_{1}}{\bar{p}_{1}} \int_{0}^{\infty} H_{\tilde{r}}(z) f(z) d z\right) \\
& \rho(201)=\rho_{0} \frac{p_{0}}{\bar{p}_{0}}\left(1-\frac{p_{1}}{\bar{p}_{1}} \int_{0}^{\infty} H_{\tilde{r}}(z) f(z) d z\right), \quad \rho(200)=\rho_{0}\left(1-\frac{p_{1}}{\bar{p}_{1}} \int_{0}^{\infty} H_{\tilde{r}}(z) f(z) d z\right) .
\end{aligned}
$$

Here $H_{\tilde{r}}(t)=\sum_{n=0}^{\infty} \tilde{R}^{*(n)}(t)$, where $\tilde{R}^{*(n)}(t)$ is n-fold convolution of the function $\tilde{R}(t)$. The constant $\rho_{0}$ is found with the help of normalization requirement.

\section{Definition of System Stationary Characteristics}

Let us define system stationary characteristics: mean sta-

$$
T_{+}=\frac{\int_{E_{+}} m(e) \rho(d e)}{\int_{E_{+}} P\left(e, E_{-}\right) \rho(d e)}, \quad T_{-}=\frac{\int_{E_{-}} m(e) \rho(d e)}{\int_{E_{+}} P\left(e, E_{-}\right) \rho(d e)} .
$$

Here $\rho(d e)$ is the EMC $\left\{\xi_{n}, n \geq 0\right\}$ stationary distribution; $m(e)$ are mean values of system dwelling times; $P\left(e, E_{-}\right)$are the probabilities of EMC $\left\{\xi_{n}, n \geq 0\right\}$ tionary operation time $T_{+}$, mean stationary restoration time $T_{-}$, stationary availability function $K_{2}$.

The sets of up states $E_{+}$and down states $E_{-}$are the following ones:

$E_{+}=\{111,211 x\}, E_{-}=\{212 x, 101 x, 202,201,200,210\}$.

Mean stationary operation time $T_{+}$and mean stationary restoration time $T_{-}$can be found with the help of Formula [6]:

transitions from up into down states.

Mean values of system dwelling times in the states are:

$$
\begin{aligned}
& m(111)=\int_{0}^{\infty} \bar{F}(t) \bar{R}(t) d t ; m(211 x)=\int_{0}^{x} \bar{R}(t) d t ; m(212 x)=m(202)=M \gamma \\
& m(201)=M \delta ; m(101 x)=x ; m(210)=m(200)=M \beta
\end{aligned}
$$


With regard to Formulas (5-7) and (9) one can define the functionals contained in (8):

$$
\begin{aligned}
& \int_{E_{+}} m(e) \rho(d e)=\rho_{0} \int_{0}^{\infty} \bar{F}(t) \bar{R}(t) d t+\rho_{0} \int_{0}^{\infty} d x \int_{0}^{x} \bar{R}(t) d t \int_{0}^{\infty} h_{\tilde{r}}(y) f(x+y) d y \\
& =\rho_{0} \int_{0}^{\infty} \bar{F}(t) \bar{R}(t) d t+\rho_{0} \int_{0}^{\infty} \bar{F}(y) d y \int_{0}^{y} h_{\tilde{r}}(y-t) \bar{R}(t) d t=\rho_{0}\left[M \alpha-\frac{p_{1}}{\bar{p}_{1}} \int_{0}^{\infty} H_{\tilde{r}}(y) \bar{F}(y) d y\right] \\
& \int_{E_{+}} P\left(e, E_{-}\right) \rho(d e)=\rho_{0}+\int_{0}^{\infty} \rho(211 x) d x=\rho_{0}\left[1+\int_{0}^{\infty} H_{\tilde{r}}(z) f(z) d z\right] \\
& \int_{E_{-}} m(e) \rho(d e)=\rho_{0} M \gamma \frac{1}{\bar{p}_{1}} \int_{0}^{\infty} d x \int_{0}^{\infty} h_{\tilde{r}}(y) f(x+y) d y+\rho_{0} \frac{1}{\bar{p}_{1}} \int_{0}^{\infty} x d x \int_{0}^{\infty} \tilde{v}(z, x) f(z) d z \\
& +\rho_{0} M \gamma \frac{1}{\bar{p}_{0}}\left(1-\frac{p_{1}}{\bar{p}_{1}} \int_{0}^{\infty} H_{\tilde{r}}(z) f(z) d z\right)+\rho_{0} M \delta \frac{p_{0}}{\bar{p}_{0}}\left(1-\frac{p_{1}}{\bar{p}_{1}} \int_{0}^{\infty} H_{\tilde{r}}(z) f(z) d z\right)+\rho_{0} M \beta\left(1-\frac{\left.p_{1} \int_{\bar{p}_{1}}^{\infty} H_{\tilde{r}}(z) f(z) d z\right)}{+\rho_{0} M \beta \frac{p_{1}}{\bar{p}_{1}} \int_{0}^{\infty} H_{\tilde{r}}(z) f(z) d z=\rho_{0} M \gamma \frac{1}{\bar{p}_{1}} \int_{0}^{\infty} H_{\tilde{r}}(y) f(y) d y+\rho_{0} \frac{1}{\bar{p}_{1}} \int_{0}^{\infty} f(z) d z \int_{0}^{\infty} x \tilde{v}(z, x) d x}\right. \\
& +\rho_{0} M \gamma \frac{1}{\bar{p}_{0}}\left(1-\frac{p_{1}}{\bar{p}_{1}} \int_{0}^{\infty} H_{\tilde{r}}(z) f(z) d z\right)+\rho_{0} M \delta \frac{p_{0}}{\bar{p}_{0}}\left(1-\frac{p_{1}}{\bar{p}_{1}} \int_{0}^{\infty} H_{\tilde{r}}(z) f(z) d z\right)+\rho_{0} M \beta=\rho_{0} M \gamma \frac{\bar{p}_{0}-p_{1} \int_{0}^{\infty} H_{\tilde{r}}(z) f(z) d z}{\bar{p}_{1}} \\
& +\rho_{0} M \delta \frac{\bar{p}_{0}-p_{1}}{\bar{p}_{1}} \int_{0}^{\infty} H_{\tilde{r}}(z) f(z) d z+\rho_{0} \frac{1}{\bar{p}_{0}} M \delta+\rho_{0} \frac{1}{\bar{p}_{0}} M \gamma+\rho_{0} M \beta-\rho_{0} M \alpha+\rho_{0} \frac{p_{1}}{\bar{p}_{1}} \int_{0}^{\infty} H_{\tilde{r}}(x) \bar{F}(x) d x .
\end{aligned}
$$

Thus, mean stationary operation time $T_{+}$is defined by the ratio

$$
T_{+}=\frac{M \alpha-\frac{p_{1}}{\bar{p}_{1}} \int_{0}^{\infty} \bar{F}(y) H_{\tilde{r}}(y) d y}{1+\int_{0}^{\infty} \bar{F}(z) d H_{\tilde{r}}(z)}
$$

and mean stationary restoration time $T_{-}$is determined by the formula:

$$
T_{-}=\frac{(M \delta+M \gamma) \frac{\bar{p}_{0}-p_{1}}{\bar{p}_{0} \bar{p}_{1}} \int_{0}^{\infty} \bar{F}(z) d H_{\tilde{r}}(z)+\frac{1}{\bar{p}_{0}}(M \delta+M \gamma)+M \beta-M \alpha+\frac{p_{1}}{\bar{p}_{1}} \int_{0}^{\infty} H_{\tilde{r}}(t) \bar{F}(t) d t}{1+\int_{0}^{\infty} \bar{F}(z) d H_{\tilde{r}}(z)}
$$

Stationary availability function is found from the ratio $K_{2}=T_{+} /\left(T_{+}+T_{-}\right)$.

We get

$K_{2}=$

$\frac{M \alpha-\frac{p_{1}}{\bar{p}_{1}} \int_{0}^{\infty} \bar{F}(y) H_{\tilde{r}}(y) d y}{(M \delta+M \gamma) \frac{\bar{p}_{0}-p_{1}}{\bar{p}_{0} \bar{p}_{1}} \int_{0}^{\infty} \bar{F}(z) d H_{\tilde{r}}(z)+\frac{1}{\bar{p}_{0}}(M \delta+M \gamma)+M \beta}$

Important characteristics for system operation quality testing are economical criteria, such as mean income $\mathrm{S}$ per unit of calendar time and mean expenses $C$ per time unit of system's up state. To define them let us use the Formula [7]:

$$
\begin{gathered}
S=\frac{\int_{E} m(e) f_{s}(e) \rho(d e)}{\int_{E} m(e) \rho(d e)} \\
C=\frac{\int_{E} m(e) f_{c}(e) \rho(d e)}{\int_{E_{+}} m(e) \rho(d e)}
\end{gathered}
$$


where $f_{s}(e), f_{c}(e)$ are the functions defining income and expenses in each state respectively.

Let $c_{1}$ be the income received per time unit of system's up state; $c_{2}$ - expenses per time unit of restoration; $c_{3}$ - expenses per time unit of control; $c_{4}$ are wastes caused by defective goods per time unit of latent failure. For the given system the functions $f_{s}(e), f_{c}(e)$ are the following:

$$
f_{s}(e)=\left\{\begin{array}{l}
c_{1}, e \in\{111,211 x\}, \\
-c_{2}, e \in\{200,210\}, \\
-c_{3}, e \in\{212 x, 202\}, \\
-c_{4}, e \in\{101 x, 201\},
\end{array} \quad f_{c}(e)=\left\{\begin{array}{l}
0, e \in\{111,211 x\} \\
c_{2}, e \in\{220,210\} \\
c_{3}, e \in\{212 x, 202\} \\
c_{4}, e \in\{101 x, 201\}
\end{array}\right.\right.
$$

With regard to (13) and (14) mean income is defined by the ratio

$$
S=\frac{M \alpha\left(c_{1}+c_{4}\right)-c_{2} M \beta-\left(c_{3} M \gamma+c_{4} M \delta\right)\left(\frac{1}{\bar{p}_{0}}+\frac{\bar{p}_{0}-p_{1}}{\bar{p}_{0} \bar{p}_{1}} \int_{0}^{\infty} \bar{F}(z) d H_{\tilde{r}}(z)\right)-\left(c_{1}+c_{4}\right) \frac{p_{1}}{\bar{p}_{1}} \int_{0}^{\infty} \bar{F}(t) H_{\tilde{r}}(t) d t}{\frac{1}{\bar{p}_{0}}(M \gamma+M \delta)+(M \gamma+M \delta) \frac{\bar{p}_{0}-p_{1}}{\bar{p}_{0} \bar{p}_{1}} \int_{0}^{\infty} \bar{F}(z) d H_{\tilde{r}}(z)+M \beta}
$$

and mean expenses are determined by the ratio

$$
C=\frac{c_{2} M \beta-c_{4} M \alpha+\left(c_{4} M \delta+c_{3} M \gamma\right) \frac{\bar{p}_{0}-p_{1}}{\bar{p}_{0} \bar{p}_{1}} \int_{0}^{\infty} \bar{F}(z) d H_{\tilde{r}}(z)+\left(c_{1}+c_{4}\right) \frac{p_{1}}{\bar{p}_{1}} \int_{0}^{\infty} \bar{F}(t) H_{\tilde{r}}(t) d t+\frac{1}{\bar{p}_{0}}\left(c_{3} M \gamma+c_{4} M \delta\right)}{M \alpha-\frac{p_{1}}{\bar{p}_{1}} \int_{0}^{\infty} \bar{F}(t) H_{\tilde{r}}(t) d t}
$$

Let us consider the case of non-random control execution periodicity $\tau>0$. Taking into account that in this case $R(t)=1(t-\tau)$, where $\tau=$ const , stationary availability function is defined by the formula

$$
K_{2}=\frac{M \alpha-\sum_{n=1}^{\infty}\left(1-\bar{p}_{1}^{n}\right) \int_{n \tau}^{(n+1) \tau} \bar{F}(t) d t}{M \beta+\frac{1}{\bar{p}_{0}}(M \gamma+\tau)+(M \gamma+\tau) \frac{\bar{p}_{0}-p_{1}}{\bar{p}_{0} \bar{p}_{1}} \sum_{n=1}^{\infty} \bar{p}_{1}^{n} \bar{F}(n \tau)}
$$

mean income can be defined in the following way

$$
S(\tau)=\frac{M \alpha\left(c_{1}+c_{4}\right)-c_{2} M \beta-\left(c_{3} M \gamma+c_{4} \tau\right) \frac{\bar{p}_{0}-p_{1}}{\bar{p}_{0} \bar{p}_{1}} \sum_{n=1}^{\infty} \bar{p}_{1}^{n} \bar{F}(n \tau)-\frac{1}{\bar{p}_{0}}\left(c_{3} M \gamma+c_{4} \tau\right)-\left(c_{1}+c_{4}\right) \sum_{n=1}^{\infty}\left(1-\bar{p}_{1}^{n}\right) \int_{n \tau}^{(n+1) \tau} \bar{F}(t) d t}{\frac{1}{\bar{p}_{0}}(M \gamma+\tau)+(M \gamma+\tau) \frac{\bar{p}_{0}-p_{1}}{\bar{p}_{0} \bar{p}_{1}} \sum_{n=1}^{\infty} \bar{p}_{1}^{n} \bar{F}(n \tau)+M \beta}
$$

mean expenses are found with the help of the ratio

$$
C(\tau)=\frac{c_{2} M \beta-c_{4} M \alpha+\left(c_{4} \tau+c_{3} M \gamma\right) \frac{\bar{p}_{0}-p_{1}}{\bar{p}_{0} \bar{p}_{1}} \sum_{n=1}^{\infty} \bar{p}_{1}^{n} \bar{F}(n \tau)+\left(c_{1}+c_{4}\right) \sum_{n=1}^{\infty}\left(1-\bar{p}_{1}^{n}\right) \int_{n \tau}^{(n+1) \tau} \bar{F}(t) d t+\frac{1}{\bar{p}_{0}}\left(c_{3} M \gamma+c_{4} \tau\right)}{M \alpha-\sum_{n=1}^{\infty}\left(1-\bar{p}_{1}^{n}\right) \int_{n \tau}^{(n+1) \tau} \bar{F}(t) d t}
$$


Let us investigate some special cases of the system operation. If errors of one kind only occur, two cases are possible.

Let $p_{0}=0, p_{0} \neq 1$, then Formulas (10-12) take the form

$$
\begin{aligned}
& T_{+}=\frac{M \alpha-\frac{p_{1}}{\bar{p}_{1}} \int_{0}^{\infty} \bar{F}(y) H_{\tilde{r}}(y) d y}{1+\int_{0}^{\infty} \bar{F}(z) d H_{\tilde{r}}(z)}, \\
& T_{-}=\frac{(M \delta+M \gamma)\left(1+\int_{0}^{\infty} \bar{F}(z) d H_{\tilde{r}}(z)\right)+M \beta-M \alpha+\frac{p_{1}}{\bar{p}_{1}} \int_{0}^{\infty} H_{\tilde{r}}(t) \bar{F}(t) d t}{1+\int_{0}^{\infty} \bar{F}(z) d H_{\tilde{r}}(z)}, K_{2}=\frac{M \alpha-\frac{p_{1}}{\bar{p}_{1}} \int_{0}^{\infty} \bar{F}(y) H_{\tilde{r}}(y) d y}{(M \delta+M \gamma)\left(1+\int_{0}^{\infty} \bar{F}(z) d H_{\tilde{r}}(z)\right)+M \beta}
\end{aligned}
$$

If $p_{1}=0, p_{0} \neq 1$, then $\tilde{r}(t)=r(t), H_{\tilde{r}}(t)=H_{r}(t)$ is renewal function generated by $\mathrm{RV}$ with $\mathrm{DF} R(t)$,

$$
\begin{aligned}
T_{+} & =\frac{M \alpha}{1+\int_{0}^{\infty} \bar{F}(z) d H_{r}(z)}, \\
T_{-} & =\frac{(M \delta+M \gamma)\left(\frac{1}{\bar{p}_{0}}+\int_{0}^{\infty} \bar{F}(z) d H_{r}(z)\right)+M \beta-M \alpha}{1+\int_{0}^{\infty} \bar{F}(z) d H_{r}(z)},
\end{aligned}
$$

$H_{r}(t)=\sum_{n=0}^{\infty} R^{*(n)}(t)$. In this case Formulas (10-12) are transformed into
In case of reliable control $\left(p_{0}=p_{1}=0\right)$ system sta-

$$
\begin{aligned}
T_{+} & =\frac{M \alpha}{1+\int_{0}^{\infty} \bar{F}(z) d H_{r}(z)}, \\
T_{-} & =\frac{(M \delta+M \gamma)\left(1+\int_{0}^{\infty} \bar{F}(z) d H_{r}(z)\right)+M \beta-M \alpha}{1+\int_{0}^{\infty} \bar{F}(z) d H_{r}(z)},
\end{aligned}
$$$$
K_{2}=\frac{M \alpha-\frac{p_{1}}{\bar{p}_{1}} \int_{0}^{\infty} \bar{F}(y) H_{r}(y) d y}{(M \delta+M \gamma)\left(1+\int_{0}^{\infty} \bar{F}(z) d H_{r}(z)\right)+M \beta}
$$

which coincide with ones found for a single-component system with reliable control.

\section{Optimization of Control Execution Periodicity}

The problem of control execution periodicity optimization is reduced to analysis of extremums of the system characteristics $K_{2}, S, C$ as functions of a single variable $\tau$.

Using Formulas (15-17), one can define an optimal period of control of the system investigated for different distribution laws of random values. The initial data for calculations of optimal values of control periodicity are: mean time of failure-free operation $M \alpha$, mean restoration time $M \beta$, control duration $M \gamma$. For example, let us suppose RV $\alpha, \beta$ and $\gamma$ to have Erlangian distribution. For the calculation of optimal value $\tau_{\text {opt }}^{s}$ providing maximal mean income $S\left(\tau_{\text {opt }}^{s}\right)$ per calendar time unit and of optimal value $\tau_{\text {opt }}^{c}$ providing minimal mean expenses $C\left(\tau_{\text {opt }}^{c}\right)$ per time unit of system's good state, the following initial data have been taken: $c_{1}=2$ c.u. $/ \mathrm{h} ; c_{2}=2$ c.u. $/ \mathrm{h} ; c_{3}=1 \mathrm{c.u} . / \mathrm{h} ; c_{4}=1 \mathrm{c.u} . / \mathrm{h}$. The results of these calculations are represented in the Table 1. The graphs of functions $K_{2}(\tau), S(\tau), C(\tau)$ for the case of Erlangian distribution of the $2^{\text {nd }}$ order and $p_{1}=0,3$, $p_{0}=0,25$ are shown in Figures 3, 4 and $\mathbf{5}$.

\section{Conclusions}

Using an apparatus of semi-Markovian processes with a common phase field it is possible to define reliability 
Table 1. Optimal control execution period definition.

\begin{tabular}{|c|c|c|c|c|c|c|c|c|c|c|c|}
\hline \multicolumn{6}{|c|}{ Initial data } & \multicolumn{6}{|c|}{ Results } \\
\hline $\begin{array}{l}\text { Distribution laws of ran- } \\
\text { dom values }\end{array}$ & $\mathrm{M} \alpha, \mathrm{h}$ & $\mathrm{M} \beta, \mathrm{h}$ & $\mathrm{M} \gamma, \mathrm{h}$ & $\mathrm{p} 1$ & P0 & $\tau_{o p t}^{k}, \mathrm{~h}$ & $K_{2}\left(\tau_{o p t}^{k}\right)$ & $\tau_{o p t}^{s}, \mathrm{~h}$ & $\begin{array}{c}S\left(\tau_{o p t}^{s}\right) \\
\text { c.u. } / \mathrm{h}\end{array}$ & $\tau_{o p t}^{c}, \mathrm{~h}$ & $C\left(\tau_{\text {opt }}^{c}\right)$, c.u. $/ \mathrm{h}$ \\
\hline Exponential & 60 & 0,5 & 0,2 & 0 & 0 & 4,833 & 0,916 & 4.833 & 1,739 & 4,833 & 0,101 \\
\hline Exponential & 60 & 0,5 & 0,2 & 0,3 & 0,25 & 5,094 & 0,867 & 5,522 & 1,568 & 48 & 1,292 \\
\hline Erlangian of the $2 \mathrm{nd}$ order & 60 & 0,5 & 0,2 & 0 & 0 & 7,744 & 0,876 & 7,744 & 1,621 & 7,744 & 0,15 \\
\hline Erlangian of the $2 \mathrm{nd}$ order & 60 & 0,5 & 0,2 & 0,3 & 0,25 & 5,911 & 0,904 & 6,302 & 1,684 & 45,535 & 0,978 \\
\hline Erlangian of the $2 \mathrm{nd}$ order & 60 & 0,5 & 0,2 & 0,2 & 0,25 & 5,007 & 0,903 & 5,259 & 1,688 & 36,112 & 0,817 \\
\hline
\end{tabular}

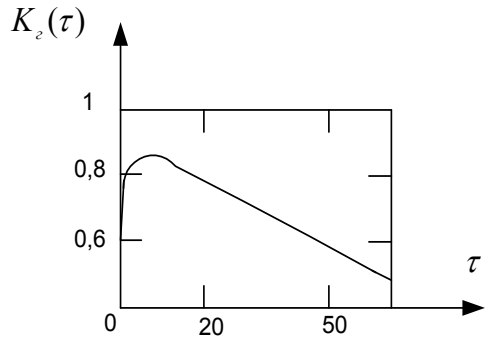

Figure 3. Graph of stationary availability function $K_{2}(\tau)$ against control periodicity $\tau$.

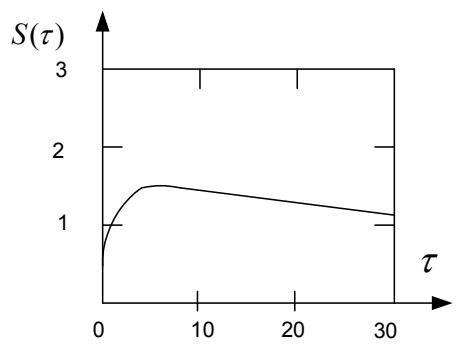

Figure 4. Graph of mean income $S(\tau)$ against control periodicity $\tau$.

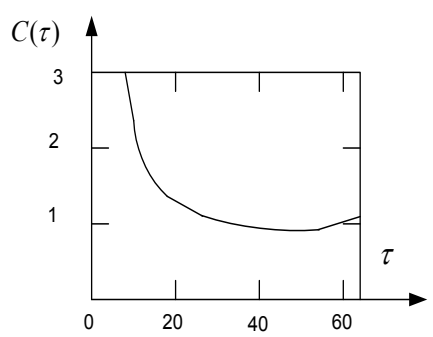

Figure 5. Graph of mean expenses $C(\tau)$ against control periodicity $\tau$. problems of control execution periodicity optimization andeconomical stationary performance indexes of restorable system with latent failures under the assumption of possibility of control errors. It allows solving the for gaining best system economical characteristics.

Later on it is planned to use the method suggested in the present article to build and investigate mathematical models of multicomponent systems and of different kinds of control.

\section{References}

[1] D. I. Cho and M. Parlar, "A Survey of Maintenance Models for Multi-Unit Systems," European Journal of Operational Research, Vol. 51, No. 2, 1991, pp. 1-23. doi:10.1016/0377-2217(91)90141-H

[2] R. Dekker and R. A. Wildeman, "A Review of MultiComponent Maintenance Models with Economic Dependence," Mathematical Methods of Operations Research, Vol. 45, No. 3, 1997, pp. 411-435. doi:10.1007/BF01194788

[3] G. N. Cherkesov, "Reliability of hardware-software complexes," Peter, St., Petersburg, 2005.

[4] A. M. Polovko and S. V. Gurov, "Reliability Theory Fundamentals," SPb: bhv-Petersburg, St. Petersburg, 2006.

[5] Yu. E. Obzherin and A. I. Peschansky, "Semi-Markovian Model of Monotonous System Maintenance with Regard to Operating Time to Failure of Each Element," Intelligent Information Management, Vol. 2, No. 8, 2010, pp. 447-456. doi:10.4236/iim.2010.28055

[6] V. S. Korolyuk and A. F. Turbin, "Markovian Restoration Processes in the Problems of System Reliability," Naukova dumka, Kiev, 1982.

[7] V. M. Shurenkov, "Ergodic Markovian Processes," Nauka, Moscow, 1989. 